\title{
Measuring wiki viability
}

\section{An empirical assessment of the social dynamics of a large sample of wikis}

\author{
Camille Roth \\ CAMS \\ CNRS/EHESS \\ 96, Bd Raspail \\ F-75006 Paris \\ France \\ camille.roth@polytechnique.edu
}

\author{
Dario Taraborelli \\ CRESS \\ Department of Sociology \\ University of Surrey \\ Guildford GU2 7XH \\ United Kingdom \\ d.taraborelli@surrey.ac.uk
}

\author{
Nigel Gilbert \\ CRESS \\ Department of Sociology \\ University of Surrey \\ Guildford GU2 7XH \\ United Kingdom \\ n.gilbert@surrey.ac.uk
}

\begin{abstract}
This paper assesses the content- and population-dynamics of a large sample of wikis, over a timespan of several months, in order to identify basic features that may predict or induce different types of fate. We analyze and discuss, in particular, the correlation of various macroscopic indicators, structural features and governance policies with specific growth patterns. While recent analyses of wiki dynamics have mostly focused on popular projects such as Wikipedia, we suggest research directions towards a more general theory of the dynamics of such communities.
\end{abstract}

\section{Categories and Subject Descriptors}

H.3.3, H.3.4 [Information storage and retrieval]: systems and software, online information services; K.4.3 [Computers and society]: collaborative work; J.4 [Computer applications]: Social and behavioral sciences-sociology

\section{General Terms}

Human Factors, Measurement, Performance

\section{Keywords}

wikis, web 2.0, online communities, governance, moderation, metrics, dynamics, viability.

\section{INTRODUCTION}

\section{Mapping the wikisphere}

Online communities have demonstrated their potential to leverage a vast amount of collaboratively contributed content. Famous examples include large open-source software development projects such as Mozilla Firefox or Linux [5] and wiki-based encyclopedias

\footnotetext{
* This work was supported by the EC-sponsored PATRES network (NEST-0 43268). We are grateful to s23.org for giving us the permission to harvest their MediaWiki statistics database.
}

Permission to make digital or hard copies of all or part of this work for personal or classroom use is granted without fee provided that copies are not made or distributed for profit or commercial advantage and that copies bear this notice and the full citation on the first page. To copy otherwise, to republish, to post on servers or to redistribute to lists, requires prior specific permission and/or a fee.

WikiSym '08, September 8-10, Porto, Portugal.

Copyright 2008 ACM 1-60558-128-3/08/09 ...\$5.00. such as Wikipedia [4]. However not all projects achieve such successful outcomes. Their destiny relies on the capacity of project maintainers to attract new members, to develop policies to secure contributors' commitment, and to promote high standards.

The question of forging a sustainable community of active contributors is particularly crucial for individuals, companies and organizations willing to launch and develop wiki-based projects. This issue relates more broadly to the understanding of the general dynamics of content-based communities and, therefore, calls for research on a wide range of wikis at various stages of development. Previous studies have mainly focused on Wikipedia. While of great interest, it seems difficult to build on this knowledge to develop a more comprehensive theory of the social structure and content dynamics of wikis-Wikipedia is a special case in many respects (population, maturity, topical range, and media attention, among other indicators). A comprehensive analysis of wiki dynamics is further hindered by the heterogeneity of wiki platforms and by the lack of tools and methods to collect data in a standardized way. Nevertheless, a first approach to generalisation can be achieved by examining a set of wikis that share the same platform.

In the following sections, we present an analysis of the evolution of a large sample of wikis based on the MediaWiki engine. In particular, we investigate some indicators that correlate with different growth rates, as a preliminary step towards a full-fledged understanding of factors that determine different patterns of evolution.

\section{Wiki ecology: demographics and viability}

The literature on the dynamics of Web-based communities is relatively scarce $[6,9]$ and, to our knowledge, the present study represents the first longitudinal analysis of the content and population dynamics of a large set of wikis over time using data retrieved via an API-based service. As well as almost always focusing on Wikipedia, previous quantitative wiki research has mainly examined the topological structure of underlying interaction or hyperlink networks [3,12] or article-level features [1,11], with little interest in the specific dynamics of the demographic determinants themselves (with the exception of [8] which investigates Wikipedia's demographics of casual vs. committed contributors). In a previous study of a static dataset describing wikis we provided a glimpse of the demographic structure of a portion of the wikisphere in terms of both population and content sizes, but stopped short of investigating its evolution.[10]

As content-based online communities, wikis most significantly vary in two dimensions: (i) contributors, who may or may not constitute an active community (as described e.g. in [2]); and (ii) pages, which may or may not amount to authoritative or useful content (as demonstrated for example by [4]). Users and pages are likely to 
obey a dual dynamic: while more users may contribute to more pages, content proliferation in turn seems to require more attention from users. As a first approximation, it may thus seem judicious to assess the healthiness of a wiki through these variables, taken as demographic proxies for its actual growth and activity.

\section{EMPIRICAL PROTOCOL}

\subsection{Dataset}

We constructed a dataset made of simple statistics gathered for a large number of MediaWiki-based wikis, ${ }^{2}$ which enabled us to consider the same set of variables across all wikis and make sure these variables were generally available. The data was collected over the period August 2007-April 2008 from a publicly-available database $^{3}$ totaling $11500+$ wikis.

\subsection{Variables}

We considered a set of four quantitative variables:

- population size $(U)$, measured by the number of registered users;

- content size $(P)$, measured by the number of so-called "good" pages, hereafter indifferently called "pages", "good pages" or "articles";

- administrator population $(A)$, or the number of users who are granted "administrator status", i.e. special rights to modify sensitive content and perform maintenance activity;

- editing activity $(E)$, measured by the total number of edits;

as well as one qualitative variable related to the presence of an optional access control mechanism: ${ }^{4}$

- editing permission $(R)$, or editing access control, i.e. the possibility of creating a page for unregistered/anonymous users. $R \in\{0,1\}$, where $1 \equiv$ "anonymous editing allowed".

These variables provide simplistic yet key indicators of the global dynamics of a wiki, and shed light on diverse aspects of its structure and evolution. We collected the values of these variables for each wiki on a daily basis and over a period of 250 days, i.e. approximatively 8 months.

\subsection{Scope restrictions}

\section{Platform exclusions}

A large number of wikis in the database are based on wikifarms, i.e. platforms hosting several wikis and providing services easing wiki creation and management. Some of these platforms return systemwide rather than wiki-specific figures when queried for wiki statistics, resulting in spurious data. To avoid this, all wikis explicitly hosted on platforms exhibiting this behavior (such as wikis hosted on wikia.com) were excluded from the dataset.

\footnotetext{
${ }^{1}$ It should be noted however that raw content growth per se may not be a good indicator of a sustainable wiki, as studies on wiki proliferation seem to suggest. [7]

${ }^{2}$ This initial dataset includes among others a large set of Wikipedias.

${ }^{3}$ Available from http://s23.org. The database is maintained by a user called "Mutante" who graciously granted us the permission to automatically harvest this data.

${ }^{4}$ This indicator was not part of the original dataset. To obtain this specific information we crawled each wiki in the dataset, at the beginning of the study, using a robot that attempted to determine whether page creation was possible without prior user registration.
}

\section{Population range}

In [10] we observed, on a similar but static dataset, that a large majority of wikis are both thinly populated (i.e. often less than 10 users) and/or do not have a significant number of pages (i.e. generally less than 10 pages). In the present study, in order to focus on a relatively homogeneous wiki population, we excluded both wikis with very large populations and those with very few users, so as to avoid basing the analysis on data spanning too many orders of magnitude in terms of population. Included in the dataset, therefore, were only wikis whose user population was within the range $[400,20000]$ on the first day of data collection (note that in this region, content sizes are widely spread, from a few to hundreds of thousands of pages).

\section{Growth discontinuities}

Some wikis experience abrupt changes of one or even several orders of magnitude in population or content size. There are many possible causes of such changes, including spam attacks, and administrative decisions to transfer, create, merge or suppress pages in bulk or admit or expel classes of user. ${ }^{5}$ To exclude wikis exhibiting such a "suspicious" behavior, on the assumption that no recruitment of users or creation of pages of a significant magnitude could "naturally" happen within a period as short as 24 hours, a threshold percentage of change $\alpha$ between two successive days, for both population and content sizes, was set. Wikis whose daily growth in either content or users was above the threshold were excluded from the dataset. $\alpha$ was set to 0.05 , i.e. wikis were excluded if they had ever experienced more than a $5 \%$ increase or decrease in users or pages over a period of 24 hours.

\section{Clean dataset}

To sum up, the final, "clean" dataset is thus made up of about 360 wikis, all of which have an initial population between 400 and 20000 users, are not hosted by 'wiki farms' that do not report useful data, and which have no major discontinuity in the daily change of their population or content.

\section{WIKI DYNAMICS}

We assessed wiki dynamics by comparing their diverse paths with respect to a set of independent variables. 'Growth' is defined in terms of population and content size change: user growth $G_{U}$ (resp. page growth $G_{P}$ ) is the ratio between final and initial populations (resp. content sizes): $G_{U}=U_{\text {last }} / U_{\text {first }}$ (resp. $G_{P}=P_{\text {last }} / P_{\text {first }}$ ).

Wiki dynamics were studied as a function of the variables listed in Section 2.2:

(i) DESCRIPTIVE INDICATORS, i.e. variables on which wiki administrators have no direct control: (a) user activity, i.e. the proportion of edits per user $(E / U)$, (b) user density, i.e. the proportion of users per page $(U / P)$, and (c) editing density, i.e. the proportion of edits per page $(E / P)$.

(ii) GOVERNANCE FACTORS, variables that wiki administrators can directly control: (a) administration ratio, i.e. the proportion of users who are granted administrator status $(A / U)$, (b) administration density, i.e. the proportion of administrators per page $(A / P)$, (c) editing permission $(R)$.

\footnotetext{
${ }^{5}$ We acknowledge that cases of spam attack are evidence that wiki sustainability is already highly damaged. However, without a method for systematically distinguishing these cases we prefer to leave aside this portion of the dataset for the sake of the present analysis.
} 


\begin{tabular}{|c|c|c|c|c|c|}
\hline \multirow{2}{*}{ Variable } & \multicolumn{5}{|c|}{ Quantile \# } \\
\hline & 1 & 2 & 3 & 4 & 5 \\
\hline edits per user & $0.14,3.67$ & $3.67,9.80$ & $9.80,24.8$ & $24.8,61.2$ & $61.2,1903$ \\
\hline admins per user & $.00169, .00192[$ & {$[.00192, .00347[$} & {$[.00347, .00576[$} & $.00576, .01[$ & $.01,1.45]$ \\
\hline edits per page & {$[0.12,9.2[$} & {$[9.2,14.3[$} & {$[14.3,21.8[$} & {$[21.8,35.1[$} & {$[35.1,47245]$} \\
\hline admins per page & {$\left[4.16 \cdot 10^{-6}, .00103[\right.$} & {$[.00103, .00309[$} & {$[.00309, .00926[$} & {$[.00926, .0299[$} & {$[.0299,2.65]$} \\
\hline \multirow[t]{2}{*}{ users per page } & {$[.00119,0.22[$} & {$[.220, .728[$} & $.728,2.07[$ & {$[2.07,7.05[$} & {$[7.05,363]$} \\
\hline & \multicolumn{5}{|c|}{ ase diagram, boundaries: $\{0.00119497, .116, .307, .619,1.24,2.44,4.99,12.1,363\}$} \\
\hline
\end{tabular}

\begin{tabular}{|c|c|c|c|c|c|}
\hline \multirow{2}{*}{ population quantiles } & & 1 & 2 & 3 & 4 \\
\hline & mean quantiles: & 400,615 & & & \begin{tabular}{|l|}
2407,19909 \\
\end{tabular} \\
\hline
\end{tabular}

Table 1: Quantile boundaries and sets

For each continuous variable, instead of carrying out a delicate analysis by dealing with clouds of points, we adopted a more insightful approach by dividing wikis into five quantiles, each including exactly $20 \%$ of all wikis in the clean dataset (see Table 1 ). We then computed and compared growth ratio means over all wikis for each quantile. Additionally, we distinguished population quantiles in order to control for user size-related effects. To this end, we plotted a growth landscape that consists of a two-dimensional representation of the various growth ratios. This representation was applied to all the above-mentioned variables, except for $R$ where there are only two "quantiles" (0 or 1$)$. For each variable except $R$, the upper graphs indicate the mean values and confidence intervals $(p<0.05)$ of each quantile on the variable considered, while the lower graphs show contour plots for the same variable with brighter areas corresponding to higher growth ratios.
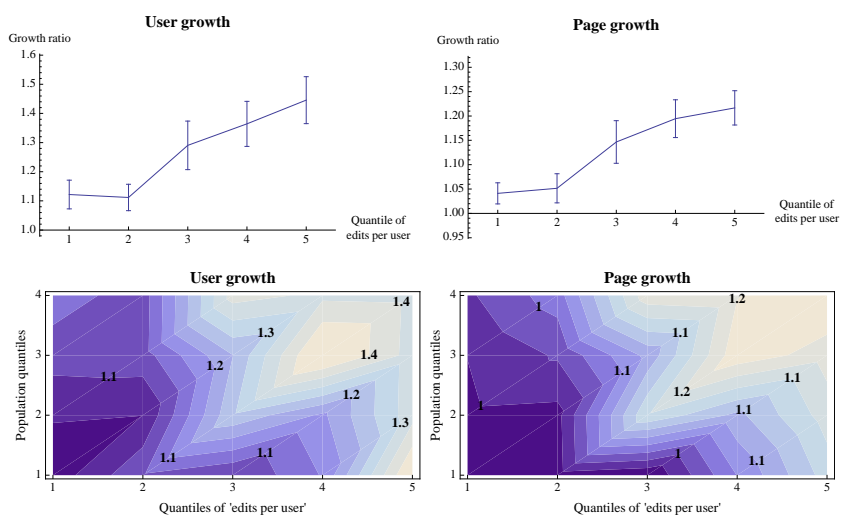

Figure 1: Growth landscape with respect to the proportion of edits per user

\subsection{Significant descriptive indicators}

We found significant correlations between a number of descriptive indicators of wiki structure and their content and population growth rates. Figure 1 shows the effect of user activity (measured as the proportion of edits per user) on growth rates. The results suggest that user activity correlates very strongly with wiki growth, not only in terms of content production (which is to a certain extent unsurprising) but also new member recruitment. The effect becomes stronger with initially more populated wikis: the more users are actively editing, the more a wiki grows in content and population. Figure 2 shows the impact of user density on growth. The results suggest that a higher number of contributors per page does not necessarily indicate mushrooming wikis: for an identical content size, we found a significant correlation between a lower number of users and higher growth ratios, both in content and new members.
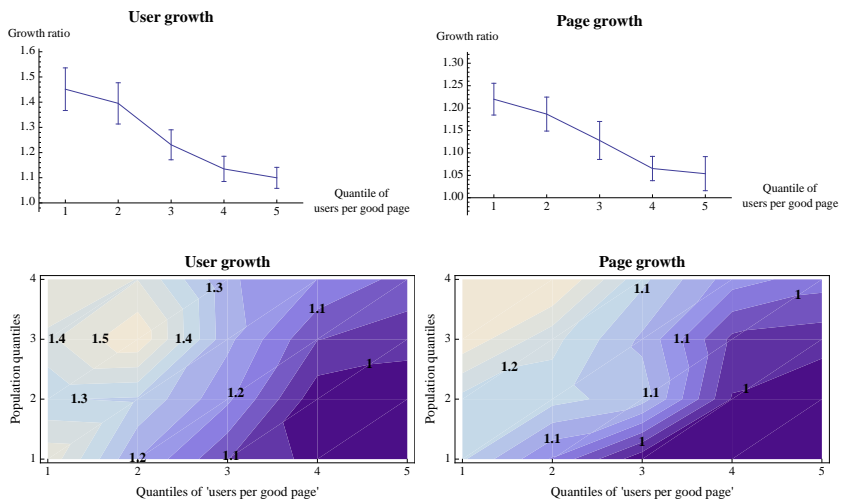

Figure 2: Growth landscape with respect to the proportion of users per good page

To better visualize the effect of user density on growth, we represented the dependent variables $G_{U}$ and $G_{P}$, independent variable $U / P$, and initial wiki positions $U_{\text {first }}$ and $P_{\text {first }}$ altogether on the same graph, yielding a phase diagram as plotted on Fig. $3 .^{6}$ In this diagram, each dot (light color) corresponds to a wiki in the database. Each arrow corresponds to a pair of quantiles "users per page, population". Widths and heights are proportional to user and content growth ratios, respectively. The size of the arrow represents the strength of the observed growth in content and population for wikis in a given region of the wikisphere.

This graph should be regarded as a map of a portion of the wikisphere, showing the expected destiny of a wiki in terms of content and population growth as a function of its initial position in the same space. This diagram broadly suggests that a wiki's position is correlated with its subsequent fate. More precisely, it illustrates that wikis in the upper/upper-right portion of the diagram are growing faster, and more interestingly it provides an overview of demographic dynamics in this region of the wikisphere.

\subsection{Effective governance factors}

Turning to governance features, we first analyzed the effects of the administrator population on wiki dynamics by looking at the overall proportion of administrators per page.

Figure 4 shows that having a relatively high number of administrators for a given content size is likely to reduce growth. There is a strong effect of the proportion of admins per page both on user and page growth. For instance, while the last quantile of admins/page ratio enjoys near-zero growth rates over 8 months, the first quantile tops overall rates $(\sim+50 \%$ for users, $\sim+25 \%$ for pages). This ef-

\footnotetext{
${ }^{6}$ For this diagram, an increased level of detail called for a larger grid, here of $8 \times 7$ quantiles; $U / P$ quantile means are represented by diagonal straight lines labelled "18 ".
} 


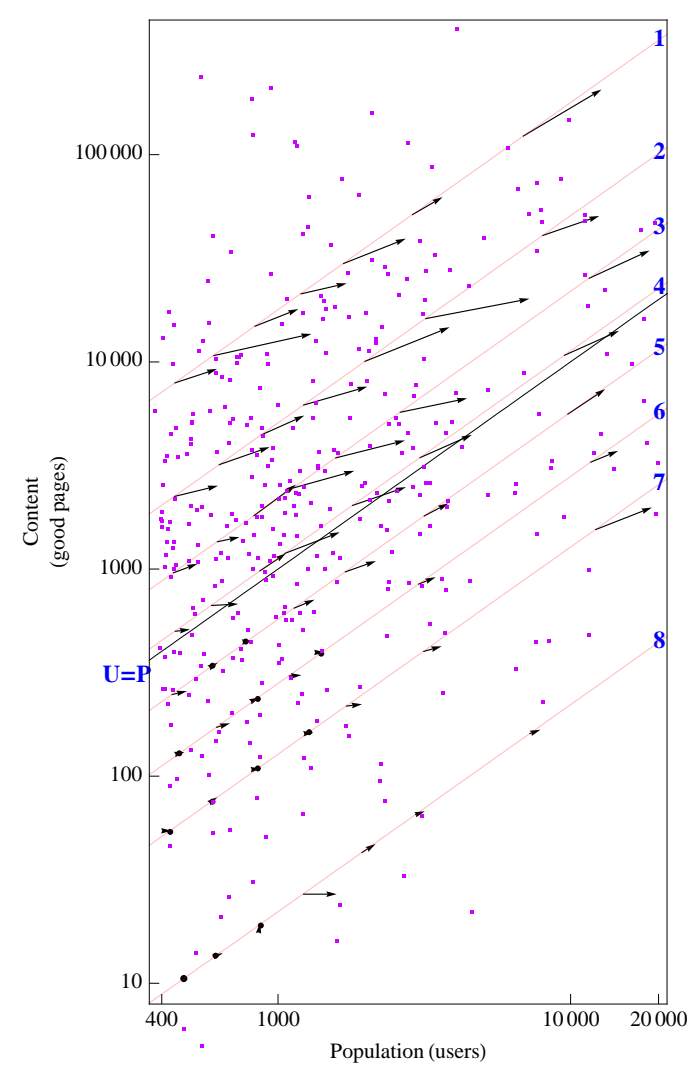

Figure 3: Phase diagram in the content/population space of wikis belonging to the clean dataset.

fect may be interpreted as the impact of strong governance activity on the proliferation of content and users.

We identified another significant effect when we considered editing permission. As a binary variable, the editing permission variable generates only two groups of wikis (wikis that allow anonymous editing versus wikis that restrict editing to registered users only). The growth landscape is consequently limited to a onedimensional comparison over population quantiles. The results in Figure 5 show that for both dimensions-population and contenthaving no access control is likely to favour growth. While a stronger page growth is quite unsurprising in wikis where no registration is required, the fact that this factor also fuels user registration is more
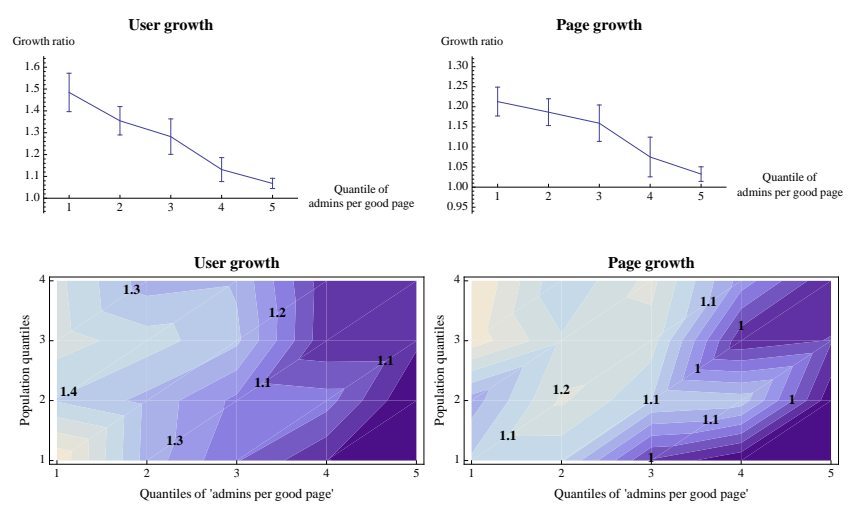

Figure 4: Growth landscape with respect to the proportion of admins per good page
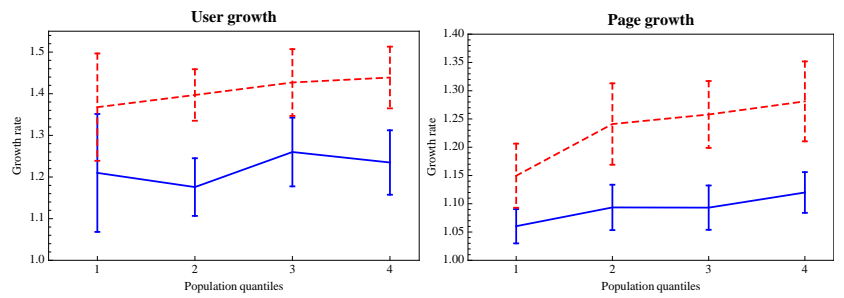

Figure 5: Growth landscape with respect to editing permission: red dashed refers to anonymously editable wikis, blue solid to wikis editable by registered users only

puzzling: one might think that if users can participate without registration, few would be inclined to register. Our results suggest that wikis with unrestricted registration seem to trigger participation more easily than wikis with relatively more restricted access.

\subsection{Neutral indicators}
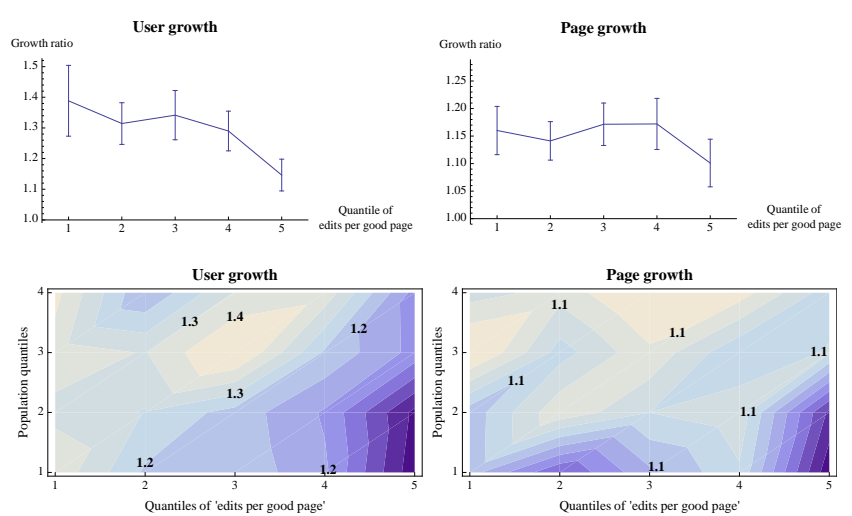

Figure 6: Growth landscape with respect to the proportion of edits per good page

Finally, we consider two indicators that showed a markedly milder correlation with wiki dynamics.

On the one hand, we found that editing density (i.e. edits/page) correlates negatively with user growth in a moderate way-with a relatively stronger effect depending on initial population sizewhile there is surprisingly no significant correlation with page growth (Figure 6).

On the other hand, higher administration ratios (i.e. admins/user) have no significant effect on content or population growth, as evidenced by the contour plot on Figure 7.

\subsection{Summary of findings}

The results of this preliminary analysis suggest that different structural and goverance-related factors have a significant effect on the dynamics of wikis, both in terms of content and population growth. Figure 8 summarizes the correlations found between growth ratios and each of the variables we considered, by comparing the gain in the population and content sizes between the last and the first quantile for each variable (variables are ranked from the most positively to the most negatively correlated). Some indicators

As far as governance factors are concerned, we observed that population growth is more than $20 \%$ larger for anonymously editable wikis. With respect to administration policies, while too many administrators per page may hinder the growth of a wiki (in terms of content size), the proportion of administrators per user 

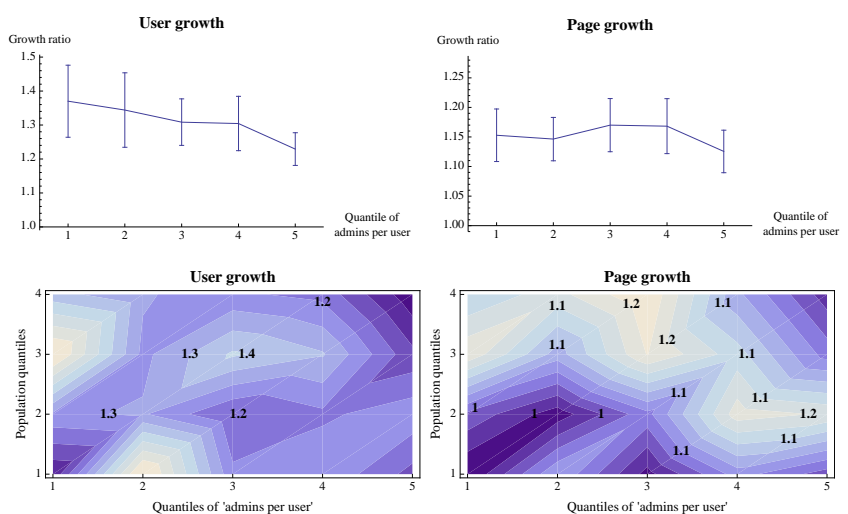

Figure 7: Growth landscape with respect to the proportion of admins per user
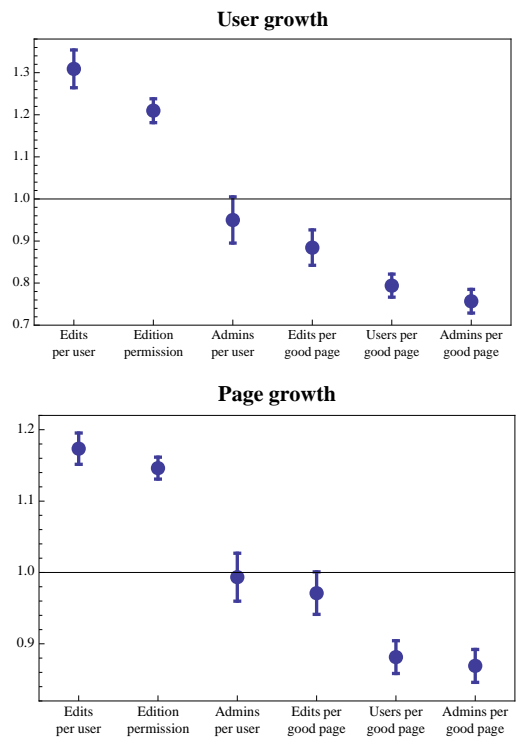

Figure 8: Comparison of growth ratios between last and first quantiles, for each variable

does not appear to show a significant influence.

\section{CONCLUDING REMARKS}

The main contribution of this study is an overview of which indicators wiki communities should take into account in order to control their demographics, by paying specific attention to some variables and consider acting upon them when possible, while neglecting others. In this respect, we drew attention to the remarkable dynamical intertwinement of population and content growth, which suggests that models of wiki dynamics will probably need to focus on the strong interrelations between these two variables.

Representing the impact of specific variables via phase diagrams can be a valuable solution for wiki administrators for monitoring purposes and for social scientists as a first step towards modeling. However, in order to develop more accurate models of wiki dynamics, richer datasets with more detailed indicators and platformindependent data collection tools need to be developed. In order to make the data tractable for this initial analysis, we restricted the dataset in several ways and a more comprehensive study, beyond the scope of the present paper, should endeavor to investigate a larger spectrum of wiki-based communities.

\section{REFERENCES}

[1] U. Brandes and J. Lerner. Visual analysis of controversy in user-generated encyclopedias. 2007.

[2] S. L. Bryant, A. Forte, and A. Bruckman. Becoming wikipedian: Transformation of participation in a collaborative online encyclopedia. In Group'05, Sanibel Island, FL, USA, Nov 6-9 2005.

[3] A. Capocci, V. Servedio, F. Colaiori, L. Buriol, D. Donato, S. Leonardi, and G. Caldarelli. Preferential attachment in the growth of social networks: the internet encyclopedia wikipedia. PRE, 74(3):036116, 2006.

[4] J. Giles. Internet encyclopaedias go head to head. Nature, 438(7070):900-901, 2005.

[5] M. W. Godfrey and Q. Tu. Evolution in open source software: A case study. ICSM, 00:131, 2000.

[6] J. Golbeck. The dynamics of web-based social networks: Membership, relationships, and change. First Monday, 12(11), November 2007.

[7] H.-J. Happel and M. Treitz. Proliferation in enterprise wikis. In Proceedings of the 8th International Conference on the Design of Cooperative Systems (COOP 08), Carry-le-Rouet, France, May 2008.

[8] A. Kittur, E. Chi, B. A. Pendleton, B. Suh, and T. Mytkowicz. Power of the few vs. wisdom of the crowd: Wikipedia and the rise of the bourgeoisie. In ALT.CHI, 2007.

[9] A. Mislove, H. S. Koppula, K. P. Gummadi, P. Druschel, and B. Bhattacharjee. Growth of the Flickr social network. In Proceedings of the 1st ACM SIGCOMM Workshop on Social Networks (WOSN'08), August 2008.

[10] C. Roth. Viable wikis: struggle for life in the wikisphere. In WikiSym '07: Proceedings of the 2007 international symposium on Wikis, pages 119-124, New York, NY, USA, 2007. ACM.

[11] D. Wilkinson and B. Huberman. Assessing the value of cooperation in Wikipedia. First Monday, 12(4), 2007.

[12] V. Zlatic, M. Bozicevic, H. Stefancic, and M. Domazet. Wikipedias: Collaborative web-based encyclopedias as complex networks. PRE, 74(1):016115, 2006. 